\title{
DETERMINATION OF THE ADEQUACY OF SPACE LAUNCH VEHICLE SYS- TEM DEVELOPMENT CONDITIONS USING A STATISTICAL SIMILARITY CRITERION
}

\author{
Yuzhnoye State Design Office \\ 3 Krivorozhskaya St., Dnipro 49008, Ukraine; e-mail: dimor9diit@gmail.com
}

This paper considers an algorithm for calculating a statistical similarity criterion for space launch vehicle (SLV) system development conditions in the form of the ratio of the dispersion ellipsoid volumes at the test stages under comparison (ground tests and full-scale tests). The criterion is calculated using the principal components method, which has some limitations in input data preparation. For example, the covariance matrix may be distorted due to large unnoticed outliers in the data or due to an inappropriate normalization, or the sample matrix may be negatively definite. To overcome these limitations in input data preparation, normalization, scaling, and the use of "complete samples" are proposed. Normalization calls for some experience in its use to account for outliers in the data so as not to get a negatively definite covariance matrix due to a nonlinear relationship between the data. Scaling is used when the input components have different dimensions, but since vectors are not invariant under scale change, the relationships between the input data may be distorted, as a consequence of which the problem of transformation of the covariance matrix into a correlation one may arise. When using "complete samples", a maximum possible fragment of the input data matrix is set off (by deleting the columns with missing parameter values or replacing a missing value of an attribute with its arithmetical mean value over the available realizations). Normalization reduces the effect of data outliers, scaling rules out a negatively definite sample matrix, and "complete samples" allow one to avoid both limitations involving source data preparation in the principal components method.

The statistical similarity criterion for SLV system development conditions, which characterizes the "proximity" of ground and flight tests, allows one to optimize the SLV development process by reducing test duration and cost.

Keywords statistical criterion, dispersion ellipsoid, principal components method, space launch vehicle, covariance matrix.

1. L. V. Krivobokov, D. V. Dunaiev, A. V. Demchenko. Estimation of the adequacy of conditions for the development of rocketry hardware as complex systems using the theory of statistical similarity (in Russian). Teh. Meh. 2017. No. 3. Pp. 64-71.

2. Iterative methods for the solution of systems of liner algebraic equations. Jacobi method (in Russian). http://bigor.bmstu.ru/?cnt/?doc=Parallel/ch030203.mod (last accessed on Jun. 8, 2018).

3. Kendall M., Stewart A. Volume 3: Multidimensional Statistical Analysis and Time Series (in Russian). Translated from English. A. N. Kolmogorov (Ed.). Moscow: Nauka, 1976. 736 pp.

4. Ayvazyan S. A., Enyukov E. S., Meshalkin L. D. Applied Statistics. Basics of Simulation and Data Preprocessing (in Russian). Moscow: Finansy i Statistika, 1983. 471 pp.

5. Kurochkina A. I. Optimal properties of the principal components of a $\lambda$-weighted covariance matrix (in Russian). In: T. V. Rubashkin, S. A. Ayvazyan, I. S. Enikov. Algorithms and Programs for Applied Statistical Analysis. Moscow: Nauka, 1980. 420 pp. 